\title{
The Relationship between Body Mass Index and Mental Health Among Iraq and Afghanistan Veterans
}

\author{
Shira Maguen, $\mathrm{PhD}^{1,2}$, Erin Madden, $\mathrm{MPH}^{7}$, Beth Cohen, MD, MAS ${ }^{1,3}$, Daniel Bertenthal, $\mathrm{MPH}^{1,4}$, \\ Thomas Neylan, $\mathrm{MD}^{1,2}$, Lisa Talbot, $P h D^{1,2}$, Carl Grunfeld, $\mathrm{MD}^{7,3}$, and Karen Seal, MD, MPH ${ }^{1,2,3}$
}

'San Francisco VA Medical Center, San Francisco, CA, USA; ${ }^{2}$ Department of Psychiatry, University of California, San Francisco, San Francisco, CA, USA; ${ }^{3}$ Department of Medicine, University of California, San Francisco, San Francisco, CA, USA; ${ }^{4}$ Mental Illness Research, Education \& Clinical Center, San Francisco, CA, USA.

BACKGROUND: Obesity is a growing public health concern and is becoming an epidemic among veterans in the post-deployment period.

OBJECTIVE: To explore the relationship between body mass index (BMI) and posttraumatic stress disorder (PTSD) in a large cohort of Iraq and Afghanistan veterans, and to evaluate trajectories of change in BMI over 3 years.

DESIGN: Retrospective, longitudinal cohort analysis of veterans' health records

PARTICIPANTS: A total of 496,722 veterans $\mathbf{( 5 9 , 7 9 0}$ female and 436,932 male veterans) whose height and weight were recorded at the Department of Veterans Affairs (VA) healthcare system at least once after the end of their last deployment and whose first post-deployment outpatient encounter at the VA was at least 1 year prior to the end of the study period (December 31, 2011).

MAIN MEASURES: BMI, mental health diagnoses. KEY RESULTS: Seventy-five percent of Iraq and Afghanistan veterans were either overweight or obese at baseline. Four trajectories were observed: "stable overweight" represented the largest class; followed by "stable obese;" "overweight/obese gaining;" and "obese losing." During the 3-year ascertainment period, those with PTSD and depression in particular were at the greatest risk of being either obese without weight loss or overweight or obese and continuing to gain weight. Adjustment for demographics and antipsychotic medication attenuated the relationship between BMI and certain mental health diagnoses. Although BMI trajectories were similar in men and women, some gender differences were observed. For example, the risk of being in the persistently obese class in men was highest for those with PTSD, whereas for women, the risk was highest among those with depression.

CONCLUSIONS: The growing number of overweight or obese returning veterans is a concerning problem for clinicians who work with these patients. Successful intervention to reduce the prevalence of obesity will require integrated efforts from primary care and mental

Electronic supplementary material The online version of this article (doi:10.1007/s11606-013-2374-8) contains supplementary material, which is available to authorized users. health to treat underlying mental health causes and assist with engagement in weight loss programs.

KEY WORDS: women's health; veteran; mental health; obesity.

J Gen Intern Med 28(Suppl 2):S563-70

DOI: $10.1007 / \mathrm{s} 11606-013-2374-8$

() Society of General Internal Medicine 2013

\section{INTRODUCTION}

Obesity is a growing public health concern and increases risk for hypertension, diabetes, metabolic syndrome, heart failure, other cardiac risk factors, and in the worst case, mortality. ${ }^{1-3}$ While individuals in the military must pass certain fitness standards before serving in the Armed Forces, there is mounting evidence that obesity is becoming an epidemic among veterans in the post-deployment period and as they age. ${ }^{3,4}$ Some studies have found that veterans experience a burst of weight gain after military discharge, which may be a particularly vulnerable time in which post-deployment eating patterns may be established. ${ }^{5}$ As these veterans return and seek medical care, it is essential that we identify and intervene with veterans who are at greatest risk for obesity before unhealthy eating patterns and weight gain occur. There is already preliminary evidence that being overweight and high body mass index (BMI) is becoming a problem for Iraq and Afghanistan veterans. ${ }^{6}$

Given that over half of Iraq and Afghanistan veterans enrolled in Department of Veterans Affairs (VA) healthcare have received one or more mental health diagnoses, with posttraumatic stress disorder (PTSD) being the most common, $^{7-9}$ it is important to better understand the association between mental health diagnoses and obesity. Among veterans, studies exploring the link between PTSD and obesity markers, such as BMI, have found mixed results, with the majority finding no association between BMI and PTSD, ${ }^{10,}{ }^{11}$ even in a sample of Iraq and Afghanistan veterans. ${ }^{12}$ While a few studies have supported the relationship between BMI and PTSD, these studies were conducted with older and predominantly male veterans. ${ }^{13,14}$ One study conducted with both men and women found a 
relationship between obesity and PTSD for women, but not for men. ${ }^{15}$ The main goal of this study was to explore the relationship between BMI and PTSD in a large cohort of Iraq and Afghanistan veterans, with particular attention to gender differences, and to evaluate trajectories of change in BMI over time. We examined BMI trajectories separately by gender because of recent studies that demonstrate gender differences in weight gain among Iraq and Afghanistan veterans. ${ }^{6}$

\section{METHODS}

\section{Study Design, Population, and Data Source}

We conducted a retrospective, longitudinal cohort study of veterans who served in Afghanistan (Operation Enduring Freedom; OEF) and Iraq (Operation Iraqi Freedom; OIF and Operation New Dawn; OND) who had used the VA healthcare system for a clinical visit between October 7, 2001 (the beginning of OEF) through December 31, 2011. We included 496,722 veterans (59,790 female and 436,932 male veterans) whose height and weight were recorded at the VA at least once after the end of their last deployment and whose first post-deployment outpatient encounter at the VA was at least 1 year prior to the end of the study period. Data for this study were derived by merging the OEF/OIF/OND Roster with three other VA national level administrative data sets: Demographics and Vital Signs (via the Corporate Data Warehouse), National Data Extract of pharmacy data via Decision Support System, and the National Patient Care Database of outpatient clinical encounters and associated clinical diagnoses. The study was approved by the Committee on Human Research, University of California, San Francisco, and the San Francisco VA Medical Center.

\section{Outcome Measures}

Body Mass Index (BMI). We calculated BMI by dividing weight in kilograms $(\mathrm{kg})$ by height in meters $(\mathrm{m})$ squared $\left(\mathrm{kg} / \mathrm{m}^{2}\right)$ for each weight measurement, using the median of each patient's recorded height measurements at all clinical visits during the study period. For our analysis, we used BMI measurements up to 3 years following the index weight measurement (after the last deployment); the average patient-level BMI was calculated for each 6 month interval starting at the index BMI measurement.

Only biologically plausible heights and weights were included in the analyses $(>70 \mathrm{lb}$ and $<700 \mathrm{lb}$; $>46 \mathrm{in}$. and $<84$ in.). To further improve data quality, all available weight measurements (both pre-deployment and postdeployment) were included in a linear mixed model of BMI over time (with a random intercept and slope for each veteran, adjusted for age and gender), in order to identify and exclude within-patient outliers (absolute value of conditional residual $\geq 10$ ). BMI measurements were retained if they were both biologically plausible and not extreme outliers $(16 \leq \mathrm{BMI} \leq 52)$. We used the International Classification of adult underweight, overweight and obesity according to BMI. ${ }^{16}$

The number and frequency of BMI measurements varied across veterans. The percentage of the cohort with BMI data during each 6-month interval subsequent to the first interval was as follows: 6-12 months: $44 \%, 12-18$ months: $40 \%$, 18-24 months: $32 \%, 24-30$ months: $28 \%$ and $30-$ 36 months: $23 \%$. Veterans with PTSD had slightly more BMI measurements recorded than veterans without PTSD. We confirmed that the mean and median index BMI did not differ for those with only one measurement compared to those with more BMI measurements.

Pregnancy-related diagnostic $\operatorname{codes}^{17}$ were used to determine whether a female veteran was pregnant at each clinical encounter. All BMI measurements in the 6 months preceding and 12 months subsequent to any clinical encounter with a pregnancy-related code were excluded from the analysis. As a result, a total of 2,061 women were completely excluded and an additional 7,623 women had some BMI values excluded.

\section{Predictor Variables}

Age at first BMI measurement and gender were extracted from the VA demographics data file. Race, marital status, and military characteristics [end of last deployment date, armed forces branch (Army, Navy/Coast Guard or Air Force), rank, component type (Reserves or Active Duty), and number of deployments (whether deployed once versus multiple times)] were extracted from the OEF/OIF/OND Roster. Distance and type of the nearest VA facility was derived from the OEF/OIF/ OND Roster by the VA Planning and System Support Group. Mental health diagnoses were identified using the International Classification of Diseases, Ninth Revision, Clinical Modification (ICD-9-CM) diagnostic codes associated with VA clinical visits, and included depression (293.83, 296.20296.25, 296.30-296.35, 300.4, and 311), PTSD (309.81), other anxiety disorders (300.00-300.09, 300.20-300.29, and 300.3), adjustment disorders (308, 309.0-309.9, excluding 309.81), alcohol use disorders (AUD; 305.00-305.03 and 303), and drug use disorders (DUD; 305.20-305.93 and 304). Veterans were also categorized by whether they had none, one, two, or three or more mental health diagnoses. Information about antidepressant medication use (including monoamine oxidase inhibitors, tricyclic antidepressants, selective serotonin reuptake inhibitors, atypical antidepressants, and serotonin-norepinephrine reuptake inhibitors) and antipsychotic medication use for greater than 30 days within each 6-month interval was obtained from VA outpatient pharmacy data. 


\section{Statistical Analysis}

We compared baseline demographic, military and clinical characteristics in female and male veterans with and without PTSD diagnoses using the chi-square test for categorical variables and the $t$-test for continuous variables.

Growth mixture modeling (GMM) is a person-centered approach to modeling longitudinal trajectories that assumes that the data are from a heterogeneous population made up of a mixture of types or "classes" of individuals (latent trajectory classes). ${ }^{18}$ This approach allows for differences in patterns of change in BMI over time where different classes of individuals are allowed to vary around different mean intercepts and slopes. For example, there may be distinct latent BMI trajectory classes, such as individuals who are slowly increasing in weight, rapidly increasing in weight, slowly losing weight, etc. The relationship between BMI trajectory and mental health conditions was allowed to vary by latent class.

We used GMM to identify latent classes of BMI trajectories and to estimate class-specific mean initial BMI (intercept) and class-specific mean change in BMI (linear and quadratic slopes). Random intercepts and slopes were included and the random effect variances were assumed to be equal across classes. Women and men were modeled separately. We specified an analysis model that included age at first post-deployment BMI measurement and race/ ethnicity as covariates for the purpose of identifying the number of trajectory classes. We selected a solution based on a combination of theory, previous research findings, ${ }^{6,19}$ model fit criteria, ${ }^{18,20,21}$ and parsimony. We performed multinomial logistic regressions using posterior probabilitybased multiple imputations ("pseudo-class" draws) to examine the association between the latent BMI trajectory classes and each mental health condition, separately. First we built models adjusted for age and race/ethnicity (as described above), and then we built models further adjusted for demographics (time from last deployment to index BMI, marital status, distance to and type of the nearest VA facility) and military characteristics (branch of service, rank, component type, and number of deployments), as well as antipsychotic medication use. We performed a sensitivity analysis that further adjusted for antidepressant medication. We also tested interactions between PTSD and age at baseline and between PTSD and race, given that both age and race are associated with BMI. Full-information maximum likelihood algorithm for handling data that is missing at random and missing completely at random was used for estimation of GMM. We assigned individuals to their most likely latent class based on posterior probabilities from the GMM models, in order to determine the probability of belonging to latent BMI trajectory class by mental health condition. Results were considered statistically significant at the $p<0.001$ level, given the large sample size. ${ }^{22,23} \mathrm{We}$ used SAS (v 9.3, SAS Institute Inc., Cary, NC) for chisquare, t-tests and linear mixed models and Mplus (v 6.12) for all GMM models. ${ }^{24}$

\section{RESULTS}

Demographic, military and clinical characteristics of the 496,722 OEF/OIF/OND veterans whose post-deployment BMI was measured at the VA are presented in Table 1 (stratified by gender and PTSD diagnosis). The cohort was comprised of 59,790 (12\%) women and 436,932 (88\%) men. The cumulative prevalence of PTSD was $30.8 \%$ among female veterans and $38.4 \%$ among male veterans. The average age at baseline was similar in men and women, except that men without PTSD were slightly older. At baseline, male veterans were more likely to fall into the overweight or obese BMI categories than female veterans ( $77 \%$ vs. $59 \%$ ).

The four latent BMI trajectory classes, which emerged independently for female and male veterans, are illustrated in Fig. 1. The trajectories were very similar in male and female veterans, although the proportion in each class differed slightly by gender. Latent class 1 ("stable overweight;" note the term overweight was chosen to describe the range of BMI for the mean values) represented the largest class, and was characterized by lower initial BMI values that slowly increased over time. Latent class 2 ("overweight/obese gaining") was characterized by initial BMI values in the overweight category with rapidly increasing weight during the first 2 years, followed by deceleration leading to slight weight loss during year three. Latent class 3 ("obese losing") represented the smallest class, and was characterized by initial BMI values in the obese category with rapid weight loss during the first 2 years, followed by accelerated weight gain and a return to obesity in year three. Latent class 4 ("stable obese") represented the second largest class, and was characterized by the highest initial BMI values and persistent obesity. The parameter estimates for the latent growth mixture models of BMI in women and men are presented in Appendix Tables 5 and 6 (available online).

The gender and mental health condition-specific distributions by BMI latent class (adjusted for age and race/ ethnicity) are presented in Table 2. In general, those with mental health conditions were more likely to belong to the three heavier BMI latent trajectory classes, i.e., the overweight/obese gaining, obese losing or stable obese classes. However, those with anxiety disorder (not including PTSD), alcohol use disorder or drug use disorder were slightly less likely to belong to the stable obese class than those without those disorders.

Overall, after adjusting for covariates, mental health conditions remained associated with increased risk of belonging to one of the three heavier BMI trajectory classes, although the magnitude of the associations were attenuated (Table 3 for women and Table 4 for men). In many cases, the association between BMI 
Table 1. Characteristics of OEF/OIF/OND Veterans Stratified by Gender and Posttraumatic Stress Disorder (PTSD)

\begin{tabular}{|c|c|c|c|c|c|}
\hline Variable & $\begin{array}{l}\text { Women } \\
\text { PTSD - } \\
(N=41,363)\end{array}$ & $\begin{array}{l}\text { Women } \\
\text { PTSD + } \\
(N=18,427)\end{array}$ & $\begin{array}{l}\text { Men PTSD - } \\
(N=\mathbf{2 6 8 , 9 9 5 )}\end{array}$ & $\begin{array}{l}\text { Men PTSD }+ \\
(N=167,937)\end{array}$ & $\begin{array}{l}\text { Total } \\
(N=496,722)\end{array}$ \\
\hline BMI at baseline & $26.7 \pm 4.84$ & $27.1 \pm 4.87$ & $28.5 \pm 4.52$ & $28.6 \pm 4.73$ & $28.4 \pm 4.67$ \\
\hline \multicolumn{6}{|l|}{ BMI category at baseline } \\
\hline Underweight (BMI: 16-18.5) & $357(0.9)$ & $186(1.0)$ & $671(0.2)$ & $474(0.3)$ & $1,688(0.3)$ \\
\hline Normal (BMI: 18.5-25) & $17,008(41.1)$ & $6,793(36.9)$ & $59,473(22.1)$ & $38,342(22.8)$ & $121,616(24.5)$ \\
\hline Overweight (BMI: 25-30) & $14,590(35.3)$ & $6,814(37.0)$ & $119,280(44.3)$ & $69,822(41.6)$ & $210,506(42.4)$ \\
\hline Obese Class I (BMI: 30-35) & $6,823(16.5)$ & $3,400(18.5)$ & $67,236(25.0)$ & $43,455(25.9)$ & $120,914(24.3)$ \\
\hline Obese Class II (BMI: 35-40) & $2,115(5.1)$ & $1,020(5.5)$ & $18,407(6.8)$ & $13,017(7.8)$ & $34,559(7.0)$ \\
\hline Obese Class III (BMI: 40+) & $470(1.1)$ & $214(1.2)$ & $3,928(1.5)$ & $2,827(1.7)$ & $7,439(1.5)$ \\
\hline Number of 6 month intervals with & $3.2 \pm 2.32$ & $4.51 \pm 2.66$ & $2.72 \pm 2.06$ & $4.1 \pm 2.52$ & $3.29 \pm 2.37$ \\
\hline \multicolumn{6}{|l|}{ BMI data during 3-year study period } \\
\hline Age at index BMI measurement (years) & $31 \pm 8.74$ & $31.1 \pm 8.56$ & $32.7 \pm 9.74$ & $30.9 \pm 8.69$ & $31.9 \pm 9.32$ \\
\hline \multicolumn{6}{|l|}{ Race } \\
\hline White & $15,649(37.8)$ & $7,151(38.8)$ & $133,168(49.5)$ & $85,723(51.0)$ & $241,691(48.7)$ \\
\hline Black & $7,914(19.1)$ & $4,099(22.2)$ & $24,727(9.2)$ & $17,196(10.2)$ & $53,936(10.9)$ \\
\hline Hispanic & $4,357(10.5)$ & $2,010(10.9)$ & $29,010(10.8)$ & $18,876(11.2)$ & $54,253(10.9)$ \\
\hline Other/Unknown & $13,443(32.5)$ & $5,167(28.0)$ & $82,090(30.5)$ & $46,142(27.5)$ & $146,842(29.6)$ \\
\hline \multicolumn{6}{|l|}{ Marital status } \\
\hline Never married & $24,473(59.3)$ & $9,988(54.5)$ & $137,520(51.2)$ & $85,967(51.3)$ & $257,948(52.0)$ \\
\hline Married & $13,267(32.2)$ & $6,416(35.0)$ & $120,247(44.8)$ & $74,777(44.6)$ & $214,707(43.3)$ \\
\hline Divorced/Separated/Widowed & $3,523(8.5)$ & $1,939(10.6)$ & $10,900(4.1)$ & $6,962(4.2)$ & $23,324(4.7)$ \\
\hline \multicolumn{6}{|l|}{ Component } \\
\hline Active Duty (vs. Reserves) & $23,589(57.0)$ & $9,984(54.2)$ & $145,389(54.0)$ & $97,527(58.1)$ & $276,489(55.7)$ \\
\hline \multicolumn{6}{|l|}{ Branch of service, } \\
\hline Army & $24,347(58.9)$ & $13,845(75.1)$ & $154,317(57.4)$ & $121,192(72.2)$ & $313,701(63.2)$ \\
\hline Air Force & $7,564(18.3)$ & $1,899(10.3)$ & $33,361(12.4)$ & $6,956(4.1)$ & $49,780(10.0)$ \\
\hline Marines & $1,573(3.8)$ & $796(4.3)$ & $37,806(14.1)$ & $30,190(18.0)$ & $70,365(14.2)$ \\
\hline Navy \& Coast Guard & $7,879(19.0)$ & $1,887(10.2)$ & $43,511(16.2)$ & $9,599(5.7)$ & $62,876(12.7)$ \\
\hline \multicolumn{6}{|l|}{ Rank } \\
\hline Enlisted (vs. Officer) & $37,560(90.8)$ & $17,228(93.5)$ & $245,819(91.4)$ & $161,833(96.4)$ & $462,440(93.1)$ \\
\hline \multicolumn{6}{|l|}{ Number of Deployments } \\
\hline Multiple (vs. Single) & $12,340(29.9)$ & $5,916(32.1)$ & $98,855(36.8)$ & $68,803(41.0)$ & $185,914(37.5)$ \\
\hline \multicolumn{6}{|l|}{ Nearest VA facility Type } \\
\hline $\begin{array}{l}\text { Community-based outpatient clinic } \\
\text { (vs. VA Medical Center) }\end{array}$ & $26,550(67.2 \%)$ & $11,774(66.4 \%)$ & $182,070(70.5 \%)$ & $116,153(71.6 \%)$ & $336,547(70.4 \%)$ \\
\hline Closest VA Facility: Distance as Crow Flies & $10.8 \pm 11.7$ & $11.1 \pm 11.3$ & $11.7 \pm 14$ & $12.3 \pm 13.8$ & $11.8 \pm 13.7$ \\
\hline Depression & $10,869(26.3)$ & $14,028(76.1)$ & $41,028(15.3)$ & $104,143(62.0)$ & $170,068(34.2)$ \\
\hline Anxiety & $7,117(17.2)$ & $8,771(47.6)$ & $32,632(12.1)$ & $64,446(38.4)$ & $112,966(22.7)$ \\
\hline Adjustment disorder & $6,425(15.5)$ & $6,166(33.5)$ & $34,964(13.0)$ & $52,175(31.1)$ & $99,730(20.1)$ \\
\hline Alcohol use disorder & $1,513(3.7)$ & $3,229(17.5)$ & $21,185(7.9)$ & $52,098(31.0)$ & $78,025(15.7)$ \\
\hline Drug use disorder & $720(1.7)$ & $1,728(9.4)$ & $8,352(3.1)$ & $26,897(16.0)$ & $37,697(7.6)$ \\
\hline \multicolumn{6}{|l|}{ Number of MH Dx } \\
\hline No $\mathrm{MH}$ diagnosis & $24,979(60.4)$ & $0(0.0)$ & $184,458(68.6)$ & $0(0.0)$ & $209,437(42.2)$ \\
\hline $1 \mathrm{MH}$ Diagnosis & $8,798(21.3)$ & $1,924(10.4)$ & $47,491(17.7)$ & $27,139(16.2)$ & $85,352(17.2)$ \\
\hline $2 \mathrm{MH}$ diagnoses & $5,383(13.0)$ & $5,523(30.0)$ & $24,302(9.0)$ & $48,534(28.9)$ & $83,742(16.9)$ \\
\hline $3+\mathrm{MH}$ diagnoses & $2,203(5.3)$ & $10,980(59.6)$ & $12,744(4.7)$ & $92,264(54.9)$ & $118,191(23.8)$ \\
\hline $\begin{array}{l}\text { Antipsychotic }>30 \text { day supply in } 6 \text { months } \\
\text { prior to index BMI measurement }\end{array}$ & $65(0.2)$ & $176(1.0)$ & $286(0.1)$ & $1,670(1.0)$ & $2,197(0.4)$ \\
\hline
\end{tabular}

BMI body mass index; OEF/OIF/OND Operation Enduring Freedom/Operation Iraqi Freedom/Operation New Dawn; MH mental health; VA Department of Veterans Affairs

trajectory class and mental health conditions remained statistically significant independent of demographic and military characteristics as well as antipsychotic medication use. After further adjusting for antidepressant medication use in a sensitivity analysis, the associations were attenuated but the direction of the relationship remained the same. Estimates for covariates in the fully-adjusted PTSD models are presented in Appendix Tables 7 and 8 (available online).

The associations between BMI latent trajectory classes and mental health conditions were similar in women and men. However, the positive association with depression was greater in women than men (see Tables 3 and 4). Also, among women, depression was the mental health condition with the highest risk of belonging to the stable obese class, but among men it was PTSD.

\section{DISCUSSION}

In this study of nearly 500,000 returning Iraq and Afghanistan veterans, we found that $75 \%$ were either overweight or obese at baseline, and most demonstrated continued weight gain over time, with $12-18 \%$ in the highest risk groups. Although rates of obesity are high in the general population, this is particularly concerning, given the young age of these veterans and the potential for future obesity-related health problems, 


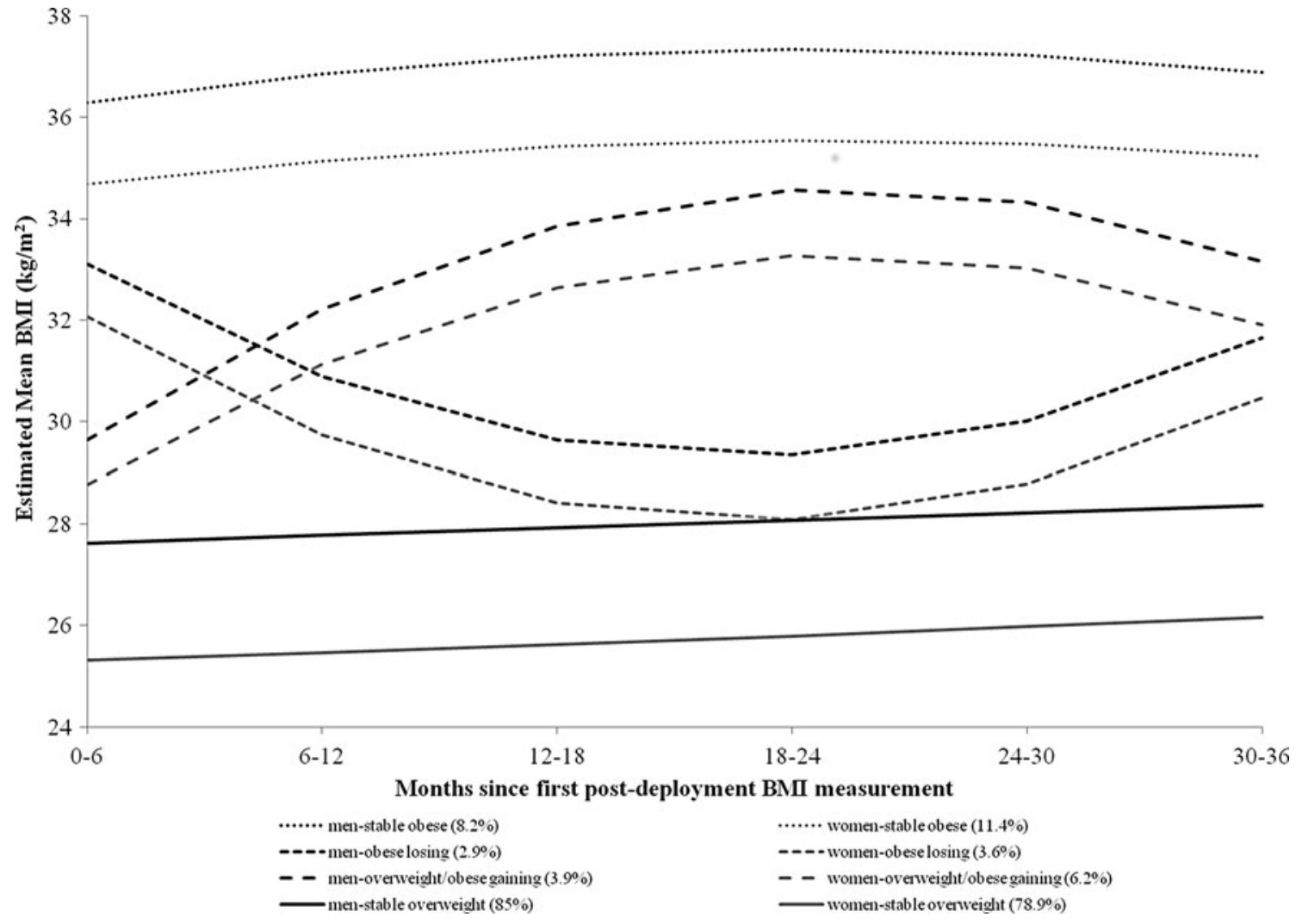

Figure 1. Trajectories of body mass index since first BMI measurement post-deployment among women and men OEF/OIF/OND veterans.

such as diabetes, heart disease, and increased mortality. Those with PTSD and depression, as well as those with multiple other mental health conditions, had the greatest likelihood of being in the highest risk groups, the stable obese and overweight/obese gaining classes. Finally, though overall BMI trajectories were similar in men and women, we observed some gender differences in the risk profile for weight gain.

These results expand upon prior work that has largely been conducted in older, male veterans. For example, those in the larger two BMI trajectories classes demonstrated gradual increases over time. We did find that two smaller

Table 2. Probability of Belonging to Latent Body Mass Index (BMI) Trajectory Class by Mental Health Condition (Column Percents Within Gender)

\begin{tabular}{|c|c|c|c|c|c|c|c|c|c|c|c|c|c|c|c|c|c|}
\hline \multirow[b]{2}{*}{$\begin{array}{l}\text { Latent } \\
\text { Class" }\end{array}$} & \multirow[b]{2}{*}{ Sex } & \multicolumn{2}{|l|}{ PTSD } & \multicolumn{2}{|c|}{ Depression } & \multicolumn{2}{|l|}{ Anxiety } & \multicolumn{2}{|c|}{ Adjustment } & \multicolumn{2}{|c|}{ Alcohol Use } & \multicolumn{2}{|c|}{ Drug Use } & \multicolumn{4}{|c|}{ Number MH Conditions } \\
\hline & & $\begin{array}{l}\text { No } \\
\text { n (\%) }\end{array}$ & $\begin{array}{l}\text { Yes } \\
\text { n (\%) }\end{array}$ & $\begin{array}{l}\text { No } \\
\text { n (\%) }\end{array}$ & $\begin{array}{l}\text { Yes } \\
\text { n (\%) }\end{array}$ & $\begin{array}{l}\text { No } \\
\text { n (\%) }\end{array}$ & $\begin{array}{l}\text { Yes } \\
\text { n (\%) }\end{array}$ & $\begin{array}{l}\text { No } \\
\text { n (\%) }\end{array}$ & $\begin{array}{l}\text { Yes } \\
\text { n (\%) }\end{array}$ & $\begin{array}{l}\text { No } \\
\text { n (\%) }\end{array}$ & $\begin{array}{l}\text { Yes } \\
\text { n (\%) }\end{array}$ & $\begin{array}{l}\text { No } \\
\text { n (\%) }\end{array}$ & $\begin{array}{l}\text { Yes } \\
\text { n (\%) }\end{array}$ & $\begin{array}{l}\text { None } \\
\text { n (\%) }\end{array}$ & $\begin{array}{l}\text { One } \\
\text { n (\%) }\end{array}$ & $\begin{array}{l}\text { Two } \\
\text { n (\%) }\end{array}$ & $\begin{array}{l}\text { Three + } \\
\text { n (\%) }\end{array}$ \\
\hline \multirow{2}{*}{$\begin{array}{l}\text { Stable } \\
\text { over- } \\
\text { weight }\end{array}$} & $\mathrm{F}$ & $\begin{array}{l}35083 \\
(84.8)\end{array}$ & $\begin{array}{l}14813 \\
(80.4)\end{array}$ & $\begin{array}{l}30130 \\
(86.3)\end{array}$ & $\begin{array}{l}19766 \\
(79.4)\end{array}$ & $\begin{array}{l}36993 \\
(84.3)\end{array}$ & $\begin{array}{l}12903 \\
(81.2)\end{array}$ & $\begin{array}{l}39539 \\
(83.8)\end{array}$ & $\begin{array}{l}10357 \\
(82.3)\end{array}$ & $\begin{array}{l}46045 \\
(83.6)\end{array}$ & $\begin{array}{l}3851 \\
(81.2)\end{array}$ & $\begin{array}{l}47926 \\
(83.6)\end{array}$ & $\begin{array}{l}1970 \\
(80.5)\end{array}$ & $\begin{array}{l}21678 \\
(86.8)\end{array}$ & $\begin{array}{l}8885 \\
(82.9)\end{array}$ & $\begin{array}{l}8763 \\
(80.4)\end{array}$ & $\begin{array}{l}10570 \\
(80.2)\end{array}$ \\
\hline & $\mathrm{M}$ & $\begin{array}{l}245644 \\
(91.3)\end{array}$ & $\begin{array}{l}146708 \\
(87.4)\end{array}$ & $\begin{array}{l}265920 \\
(91.1)\end{array}$ & $\begin{array}{l}126432 \\
(87.1)\end{array}$ & $\begin{array}{l}306744 \\
(90.3)\end{array}$ & $\begin{array}{l}85608 \\
(88.2)\end{array}$ & $\begin{array}{l}315284 \\
(90.1)\end{array}$ & $\begin{array}{l}77068 \\
(88.4)\end{array}$ & $\begin{array}{l}327323 \\
(90.0)\end{array}$ & $\begin{array}{l}65029 \\
(88.7)\end{array}$ & $\begin{array}{l}361233 \\
(89.9)\end{array}$ & $\begin{array}{l}31119 \\
(88.3)\end{array}$ & $\begin{array}{l}169620 \\
(92.0)\end{array}$ & $\begin{array}{l}66946 \\
(89.7)\end{array}$ & $\begin{array}{l}63985 \\
(87.8)\end{array}$ & $\begin{array}{l}91801 \\
(87.4)\end{array}$ \\
\hline \multirow{4}{*}{$\begin{array}{l}\text { Over- } \\
\text { weight/ } \\
\text { obese } \\
\text { gaining } \\
\text { Obese } \\
\text { losing }\end{array}$} & $\mathrm{F}$ & $\begin{array}{l}1116 \\
(2.7)\end{array}$ & $\begin{array}{l}1019 \\
(5.5)\end{array}$ & $\begin{array}{l}796 \\
(2.3)\end{array}$ & $\begin{array}{l}1339 \\
(5.4)\end{array}$ & $\begin{array}{l}1298 \\
(3.0)\end{array}$ & $\begin{array}{l}837 \\
(5.3)\end{array}$ & $\begin{array}{l}1481 \\
(3.1)\end{array}$ & $\begin{array}{l}654 \\
(5.2)\end{array}$ & $\begin{array}{l}1841 \\
(3.3)\end{array}$ & $\begin{array}{l}294 \\
(6.2)\end{array}$ & $\begin{array}{l}1966 \\
(3.4)\end{array}$ & $\begin{array}{l}169 \\
(6.9)\end{array}$ & $\begin{array}{l}459 \\
(1.8)\end{array}$ & $\begin{array}{l}365 \\
(3.4)\end{array}$ & $\begin{array}{l}491 \\
(4.5)\end{array}$ & $\begin{array}{l}820 \\
(6.2)\end{array}$ \\
\hline & $\mathrm{M}$ & $\begin{array}{l}3642 \\
(1.4)\end{array}$ & $\begin{array}{l}5336 \\
(3.2)\end{array}$ & $\begin{array}{l}4001 \\
(1.4)\end{array}$ & $\begin{array}{l}4977 \\
(3.4)\end{array}$ & $\begin{array}{l}5740 \\
(1.7)\end{array}$ & $\begin{array}{l}3238 \\
(3.3)\end{array}$ & $\begin{array}{l}6345 \\
(1.8)\end{array}$ & $\begin{array}{l}2633 \\
(3.0)\end{array}$ & $\begin{array}{l}6431 \\
(1.8)\end{array}$ & $\begin{array}{l}2547 \\
(3.5)\end{array}$ & $\begin{array}{l}7510 \\
(1.9)\end{array}$ & $\begin{array}{l}1468 \\
(4.2)\end{array}$ & $\begin{array}{l}1795 \\
(1.0)\end{array}$ & $\begin{array}{l}1364 \\
(1.8)\end{array}$ & $\begin{array}{l}1881 \\
(2.6)\end{array}$ & $\begin{array}{l}3938 \\
(3.8)\end{array}$ \\
\hline & $\mathrm{F}$ & $\begin{array}{l}800 \\
(1.9)\end{array}$ & $\begin{array}{l}541 \\
(2.9)\end{array}$ & $\begin{array}{l}595 \\
(1.7)\end{array}$ & $\begin{array}{l}746 \\
(3.0)\end{array}$ & $\begin{array}{l}865 \\
(2.0)\end{array}$ & $\begin{array}{l}476 \\
(3.0)\end{array}$ & $\begin{array}{l}1017 \\
(2.2)\end{array}$ & $\begin{array}{l}324 \\
(2.6)\end{array}$ & $\begin{array}{l}1183 \\
(2.1)\end{array}$ & $\begin{array}{l}158 \\
(3.3)\end{array}$ & $\begin{array}{l}1226 \\
(2.1)\end{array}$ & $\begin{array}{l}115 \\
(4.7)\end{array}$ & $\begin{array}{l}385 \\
(1.5)\end{array}$ & $\begin{array}{l}223 \\
(2.1)\end{array}$ & $\begin{array}{l}315 \\
(2.9)\end{array}$ & $\begin{array}{l}418 \\
(3.2)\end{array}$ \\
\hline & $\mathrm{M}$ & $\begin{array}{l}3216 \\
(1.2)\end{array}$ & $\begin{array}{l}3625 \\
(2.2)\end{array}$ & $\begin{array}{l}3590 \\
(1.2)\end{array}$ & $\begin{array}{l}3251 \\
(2.2)\end{array}$ & $\begin{array}{l}4759 \\
(1.4)\end{array}$ & $\begin{array}{l}2082 \\
(2.1)\end{array}$ & $\begin{array}{l}5118 \\
(1.5)\end{array}$ & $\begin{array}{l}1723 \\
(2.0)\end{array}$ & $\begin{array}{l}5132 \\
(1.4)\end{array}$ & $\begin{array}{l}1709 \\
(2.3)\end{array}$ & $\begin{array}{l}5675 \\
(1.4)\end{array}$ & $\begin{array}{l}1166 \\
(3.3)\end{array}$ & $\begin{array}{l}1808 \\
(1.0)\end{array}$ & $\begin{array}{l}1070 \\
(1.4)\end{array}$ & $\begin{array}{l}1405 \\
(1.9)\end{array}$ & $\begin{array}{l}2558 \\
(2.4)\end{array}$ \\
\hline \multirow[t]{2}{*}{$\begin{array}{l}\text { Stable } \\
\text { obese }\end{array}$} & $\mathrm{F}$ & $\begin{array}{l}4364 \\
(10.6)\end{array}$ & $\begin{array}{l}2054 \\
(11.1)\end{array}$ & $\begin{array}{l}3372 \\
(9.7)\end{array}$ & $\begin{array}{l}3046 \\
(12.2)\end{array}$ & $\begin{array}{l}4746 \\
(10.8)\end{array}$ & $\begin{array}{l}1672 \\
(10.5)\end{array}$ & $\begin{array}{l}5162 \\
(10.9)\end{array}$ & $\begin{array}{l}1256 \\
(10.0)\end{array}$ & $\begin{array}{l}5979 \\
(10.9)\end{array}$ & $\begin{array}{l}439 \\
(9.3)\end{array}$ & $\begin{array}{l}6224 \\
(10.9)\end{array}$ & $\begin{array}{l}194 \\
(7.9)\end{array}$ & $\begin{array}{l}2457 \\
(9.8)\end{array}$ & $\begin{array}{l}1249 \\
(11.6)\end{array}$ & $\begin{array}{l}1337 \\
(12.3)\end{array}$ & $\begin{array}{l}1375 \\
(10.4)\end{array}$ \\
\hline & $\mathrm{M}$ & $\begin{array}{l}16493 \\
(6.1)\end{array}$ & $\begin{array}{l}12268 \\
(7.3)\end{array}$ & $\begin{array}{l}18250 \\
(6.3)\end{array}$ & $\begin{array}{l}10511 \\
(7.2)\end{array}$ & $\begin{array}{l}22611 \\
(6.7)\end{array}$ & $\begin{array}{l}6150 \\
(6.3)\end{array}$ & $\begin{array}{l}23046 \\
(6.6)\end{array}$ & $\begin{array}{l}5715 \\
(6.6)\end{array}$ & $\begin{array}{l}24763 \\
(6.8)\end{array}$ & $\begin{array}{l}3998 \\
(5.5)\end{array}$ & $\begin{array}{l}27265 \\
(6.8)\end{array}$ & $\begin{array}{l}1496 \\
(4.2)\end{array}$ & $\begin{array}{l}11235 \\
(6.1)\end{array}$ & $\begin{array}{l}5250 \\
(7.0)\end{array}$ & $\begin{array}{l}5565 \\
(7.6)\end{array}$ & $\begin{array}{l}6711 \\
(6.4)\end{array}$ \\
\hline
\end{tabular}

*Latent trajectory classes were determined from gender-specific latent growth mixture models of BMI that included age and race as covariates. Individuals were assigned to the latent BMI trajectory class for which they had the highest posterior probability of belonging

MH mental health; PTSD posttraumatic stress disorder 
Table 3. Associations Between Body Mass Index (BMI) Trajectory Classes and Mental Health (MH) Conditions Among Women Veterans $(N=\mathbf{5 9 , 7 9 0 )}$

\begin{tabular}{|c|c|c|c|c|c|c|}
\hline \multirow[b]{2}{*}{ MH Condition } & \multicolumn{2}{|c|}{$\begin{array}{l}\text { Overweight/obese gaining vs. } \\
\text { Stable overweight }\end{array}$} & \multicolumn{2}{|c|}{ Obese losing vs. Stable overweight } & \multicolumn{2}{|c|}{ Stable obese vs. Stable overweight } \\
\hline & $\begin{array}{l}\text { Age and Race } \\
\text { AOR }^{*}(95 \% \text { CI })\end{array}$ & $\begin{array}{l}\text { Fully AOR } \dagger \\
(95 \% \text { CI })\end{array}$ & $\begin{array}{l}\text { Age and Race } \\
\text { AOR }^{*}(95 \% \text { CI })\end{array}$ & $\underset{\mathrm{CI})}{\mathrm{AOR} \dagger}$ & $\begin{array}{l}\text { Age and Race } \\
\text { AOR }^{*}(95 \% \text { CI })\end{array}$ & $\begin{array}{l}\text { Fully AOR } \uparrow \\
(95 \% \text { CI })\end{array}$ \\
\hline TSD & $56)^{*}$ & $41)^{*}$ & 31 & 1.21 & 1.11 & 1.13 \\
\hline ep & & ק & $9)^{ \pm}$ & & & \\
\hline Anx & $.39)^{ \pm}$ & $5(1.05,1.27)$ & $9(1.07,1.33)$ & $1.12(1,1$ & $5,1.09)$ & $0.99(0.92,1.06)$ \\
\hline Adjustment & $1.24(1.13,1.37)^{\star}$ & $1.19(1.08,1.32)$ & $0.99(0.86,1.13)$ & $1.03(0.9,1.18)$ & $0.96(0.89,1.04)$ & $1.03(0.96,1.12)$ \\
\hline Alcohol use & $1.39(1.21,1.6)^{\frac{x}{2}}$ & $1.16(1.01,1.35)$ & $1.26(1.06,1.51)$ & $1.18(0.99,1.41)$ & $0.91(0.81,1.02)$ & $0.87(0.76,0.99)$ \\
\hline Drug & $1.47(1.24,1.75)^{*}$ & $1.12(0.93,1.35$ & $1.69(1.38,2.07)^{\$}$ & $1.48(1.19,1.85)^{*}$ & $0.8(0.68,0.95)$ & $0.71(0.6,0.85)^{2}$ \\
\hline $1 \mathrm{MH}$ condition & $1.23(1.08,1.4)$ & $1.19(1.04,1.36)$ & $1.13(0.97,1.32)$ & $1.13(0.96,1.33)$ & $1.23(1.12,1.34)$ & $1.21(1.1,1.32)^{\star}$ \\
\hline $2 \mathrm{MH} \mathrm{co}$ & $1.43(1.27,1.62)^{\ddagger}$ & $1.34(1.18,1.51)^{\ddagger}$ & & & & $1.34(1.22,1.46)^{\ddagger}$ \\
\hline $3+\mathrm{MH}$ conditions & 1.9 & $1.47(1.31,1.65$ & $.21,1.58)^{*}$ & $1.34(1.1$ & $1.17(1.07,1.27$ & $1.17(1.07$ \\
\hline
\end{tabular}

* Odds ratios adjusted for age at first post-deployment BMI measurement and race

${ }^{\dagger}$ Odds ratios further adjusted for marital status, rank, branch of service, component, multiple vs. single deployments, time since end of last deployment, type and distance of nearest VA facility, and time on antipsychotic medication(s)

${ }^{*} p<0.001$

classes, "overweight/obese gaining" and "obese losing," reversed direction around 18-24 months. One possibility is that these weight changes coincide with the time that veterans with mental health problems engage in minimally adequate mental health treatment; ${ }^{25}$ for some, this may be a weight loss facilitator, while for others, it may be a temporary barrier as potentially difficult emotions are experienced. Our finding that those with mental health conditions were more likely to be in the overweight or obese groups, with some exceptions (e.g., alcohol use disorders) is also consistent with earlier work. ${ }^{13,14}$ However, our finding that men and women have similar BMI trajectories deviates somewhat from past studies of Iraq and Afghanistan veterans, ${ }^{6}$ but is similar to population-based studies. $^{26}$

We also found that depression was the mental health condition with the highest risk of belonging to the stable obese class among women, but among men, the highest risk was found in those with PTSD. This is contrary to a study of community dwelling adolescents and young adults in Germany, which found a relationship between obesity and PTSD for women, but not for men. ${ }^{15}$ The different gender effects in our veteran population have important treatment implications, given the growing number of women in the military and the fact that depression is the most common diagnosis among recently returning female veterans seeking VA services. ${ }^{22}$ For example, primary care providers working with depressed female veterans can screen for problematic eating behaviors in this group, assess motivation for behavior change, and enlist the assistance of mental health colleagues to directly address problematic eating leading to weight gain.

The relationship between particular mental health diagnoses and gender highlights the need to monitor weight, refer patients with mental health and weight issues for specialty mental health care, and potentially develop weight management interventions within mental health clinics. This also underscores the need for collaborative care efforts among primary care and mental health clinicians. Interventions that take both mental health components and weight status into account may best facilitate weight loss, and programs that do not account for mental health status may be less effective. ${ }^{27,28}$

Table 4. Associations Between Body Mass Index (BMI) Trajectory Classes and Mental Health (MH) Conditions Among Men Veterans ( $N=$ 436,932)

\begin{tabular}{|c|c|c|c|c|c|c|}
\hline \multirow[b]{2}{*}{ MH Condition } & \multicolumn{2}{|c|}{$\begin{array}{l}\text { Overweight/obese gaining vs. Stable } \\
\text { overweight }\end{array}$} & \multicolumn{2}{|c|}{ Obese losing vs. Stable overweight } & \multicolumn{2}{|c|}{ Stable obese vs. Stable overweight } \\
\hline & $\begin{array}{l}\text { Age and Race } \\
\operatorname{AOR}^{*}(95 \% \mathrm{CI})\end{array}$ & $\begin{array}{l}\text { Fully AOR } \dagger \\
(95 \% \text { CI })\end{array}$ & $\begin{array}{l}\text { Age and Race } \\
\text { AOR }^{*}(95 \% \mathrm{CI})\end{array}$ & $\begin{array}{l}\text { Fully AOR } \dagger \\
(95 \% \text { CI })\end{array}$ & $\begin{array}{l}\text { Age and Race } \\
\operatorname{AOR}^{*}(95 \% \mathrm{CI})\end{array}$ & $\begin{array}{l}\text { Fully AOR } \dagger \\
(95 \% \text { CI })\end{array}$ \\
\hline PTS & & & 1) & )$^{*}$ & $22+22+2+2$ & 1.1 \\
\hline & & & & 1.10 & & \\
\hline An & $31(1.25,1.37)$ & $1.15(1.1,1.2)^{\ddagger}$ & $1.13(1.08,1.19)^{*}$ & $1.07(1.02,1.13)$ & $0.98(0.95,1.01)$ & $0.95(0.92,0.98)$ \\
\hline $\mathrm{Ad}$ & $1.27)^{*}$ & $1.13(1.08,1.19)^{*}$ & 1. & 1.09 & $.99,1.06)$ & $1.04(1,1.07)$ \\
\hline & $1.44)^{\ddagger}$ & $1.12(1.07 .1 .18)^{*}$ & $1.29)^{\ddagger}$ & & $0.86(0.83,0.89)^{\ddagger}$ & $0.81(0.77,0.84)$ \\
\hline Drug $u$ & $1.46(1.37,1.55)^{\ddagger}$ & $1.1(1.03,1.17)$ & $1.54(1.45,1.65)^{\ddagger}$ & $1.34(1.25,1.43)^{*}$ & $0.67(0.63,0.71)$ & $0.6(0.57,0.64)^{7}$ \\
\hline $1 \mathrm{MH}$ & (112 127 & $1.12(1.05,1.19)^{*}$ & $1.15(1.08,1.23)^{\star}$ & $1.1(1.02,1.17)$ & $1.16(1.12,1.21)^{\ddagger}$ & $1.11(1.07,1.15)^{*}$ \\
\hline $2 \mathrm{~N}$ & 1.3 & $1.22(1.15,1.29)^{*}$ & $1.26(1.19 .1 .35)^{\ddagger}$ & $1.19(1.11 .1 .27)^{\ddagger}$ & $1.27(1.23,1.32)^{*}$ & $1.19(1.14 .1 .2$ \\
\hline $3+\mathrm{MH}$ conditions & $1.65(1.57,1.73)^{*}$ & $1.32(1.25,1.4)^{t}$ & $1.36(1.29,1.44)^{*}$ & $1.24(1.17,1.32)^{*}$ & $1.1(1.06,1.14)^{4}$ & $1.02(0.98,1.06)$ \\
\hline
\end{tabular}

*Odds ratios adjusted for age at baseline and race

${ }^{+}$Odds ratios further adjusted for marital status, rank, branch of service, component, multiple vs. single deployments, time since end of last deployment, type and distance of nearest VA facility, and time on antipsychotic medication(s)

${ }^{t} p<0.001$ 
Managing overweight or obese patients with mental health problems within the VA can be effectively accomplished within existing systems of care, particularly with the nationwide implementation of primary-care mental health integration programs. For veterans seen outside the VA system, coordinated primary and mental healthcare is also important. Ensuring that barriers to weight loss and adoption of healthy choices are addressed from an integrated care perspective is critical in VA and non-VA settings. For example, providers can send consistent messages about adverse health reactions and work together on amelioration of mental health problems, such as depression and PTSD. While referral to VA weight loss programs such as MOVE! is an important step, assisting veterans with barriers to attending, completing, and benefitting from these programs can be accomplished through primary care and mental health co-management, and further through engaging programs such as VA Telephone Lifestyle Coaching available through the VA. For example, if a barrier to attending MOVE! has to do with the fact that the veteran engages in overeating to cope with traumatic memories or depressive thoughts, these might need to be addressed within an integrated care setting before a veteran can benefit from a weight loss program.

There are several reasons that treating underlying mental health issues may facilitate weight loss. First, individuals with mental health problems such as PTSD and depression may engage in unhealthy eating in response to stress or to assist with emotional regulation. ${ }^{29,}{ }^{30}$ For example, eating may serve as a coping strategy for those with depressed mood or anxiety the same way that alcohol and/or drugs may serve as a coping strategy for those with substance use disorders. Consequently, treating the underlying mental health cause of the weight gain is critical. Unfortunately, a large number of returning veterans with mental health problems do not engage in adequate mental health care. ${ }^{25,31}$ Our study suggests that efforts to improve engagement and retention in mental health care may improve the mental and physical health of returning veterans. ${ }^{32}$

There are several limitations of our work. First, veterans returning from recent deployments may have higher BMI due to muscle mass rather than truly being overweight or obese, and we were not able to include measurements of body fat or other more specific indicators of obesity. Second, the number of BMI measures differed across our subjects. Third, rates of PTSD may be greater in our cohort, given that these veterans all sought primary care within the VA. Fourth, mental health conditions were assessed by diagnostic codes rather than clinical interviews. Finally, given that we used administrative data, our analyses measured associations, rather than cause-and-effect relationships, between mental health and BMI trends.

Despite these limitations, our findings have important implications for future research and for clinical care of returning veterans. Though overall BMI trajectories did not differ in men and women, we did find some gender differences in associations of mental health conditions and BMI, underscoring efforts to provide gender specific care for returning veterans. ${ }^{33}$ In addition, the fact that $75 \%$ of returning veterans were overweight and obese and that those with mental health diagnoses had increased obesity risk highlights the need for all providers to counsel returning veterans about weight, and for efforts to better integrate primary and mental health care.

Acknowledgements: This research was supported by Department of Defense Concept Award Grant (Maguen), VA Health Sciences Research and Development (HSR\&D) Career Development Award (Maguen), National Institutes of Health grant K23 HL 094765-01 (Cohen), and the Mental Illness Research and Education Clinical Center of the US Veterans Health Administration.

The views expressed in this article are those of the authors and do not necessarily reflect the position or policy of the Department of Veterans Affairs or the U.S. government.

Conflict of Interest: The authors do not have any competing interest to report. Dr. Maguen had full access to all the data in the study and takes responsibility for the integrity of the data and the accuracy of the data analysis.

Corresponding Author: Shira Maguen, PhD; San Francisco VA Medical Center, 4150 Clement Street (116-P), San Francisco, CA 94121, USA (e-mail: Shira.Maguen@va.gov).

\section{REFERENCES}

1. Kenchaiah S, Evans JC, Levy D, et al. Obesity and the risk of heart failure. N Engl J Med. 2002;5:305-313.

2. Adams KF, Schatzkin A, Harris TB, et al. Overweight, obesity, and mortality in a large prospective cohort of persons 50 to 71 years old. N Engl J Med. 2006;8:763-778.

3. Nelson KM. The burden of obesity among a national probability sample of veterans. J Gen Intern Med. 2006;9:915-919.

4. Das SR, Kinsinger LS, Yancy WS Jr, et al. Obesity prevalence among veterans at Veterans Affairs medical facilities. Am J Prev Med. 2005;3:291-294.

5. Koepsell TD, Littman AJ, Forsberg CW. Obesity, overweight, and their life course trajectories in veterans and non-veterans. Obesity. 2012;2:434-439.

6. Rosenberger PH, Ning Y, Brandt C, Allore H, Haskell S. BMI trajectory groups in veterans of the Iraq and Afghanistan wars. Prev Med. 2011;3:149-154.

7. Epidemiology Program, Post-Deployment Health Group, Office of Public Health, Veterans Health Administration, Department of Veterans Affairs. (2012). Analysis of VA Health Care Utilization among Operation Enduring Freedom, Operation Iraqi Freedom, and Operation New Dawn Veterans, from 1st Btr FY 2002 through 3rd Qtr FY 2012. Washington, DC: Author. Retrieved from (URL) http://www.publichealth.va.gov/docs/epidemiology/ healthcare-utilization-report-fy2012-qtr3.pdf.

8. Hoge CW, Castro CA, Messer SC, McGurk D, Cotting DI, Koffman RL. Combat duty in Iraq and Afghanistan, mental health problems, and barriers to care. N Engl J Med. 2004;1:13-22.

9. Seal KH, Bertenthal D, Miner CR, Sen S, Marmar C. Bringing the war back home: mental health disorders among 103,788 US veterans returning from Iraq and Afghanistan seen at Department of Veterans Affairs facilities. Arch Intern Med. 2007;5:476-482.

10. Vieweg WV, Julius DA, Benesek J, et al. Posttraumatic stress disorder and body mass index in military veterans. Preliminary findings. Prog Neuropsychopharmacol Biol Psychiatry. 2006;6:1150-1154.

11. Kozaric-Kovacic D, Ilic MG, Romic Z, Vidovic A, Jendricko T, Pivac N. Body mass index in male Caucasian veterans with or without 
posttraumatic stress disorder. Prog Neuropsychopharmacol Biol Psychiatry. 2009;8:1447-1450.

12. Barber J, Bayer L, Pietrzak RH, Sanders KA. Assessment of rates of overweight and obesity and symptoms of posttraumatic stress disorder and depression in a sample of Operation Enduring Freedom/Operation Iraqi Freedom veterans. Mil Med. 2011;2:151-155.

13. Vieweg WV, Julius DA, Bates J, et al. Posttraumatic stress disorder as a risk factor for obesity among male military veterans. Acta Psychiatr Scand. 2007;6:483-487.

14. Coughlin SS, Kang HK, Mahan CM. Selected health conditions among overweight, obese, and non-obese veterans of the 1991 Gulf War: results from a survey conducted in 2003-2005. Open Epidemiol J. 2011;4:140-146.

15. Perkonigg A, Owashi T, Stein MB, Kirschbaum C, Wittchen HU. Posttraumatic stress disorder and obesity: evidence for a risk association. Am J Prev Med. 2009;1:1-8.

16. World Health Organization. BMI classification. In Global Database on Body Mass Index. Retrieved June 29, 2012, from http://apps.who.int/ bmi/index.jsp?introPage=intro_3.html.

17. Mattocks KM, Skanderson M, Goulet $\mathbf{J L}$, et al. Pregnancy and mental health among women veterans returning from Iraq and Afghanistan. J Womens Health. 2010;19:2159-2166.

18. Muthén B. Latent variable analysis: Growth mixture modeling and related techniques for longitudinal data. In: Kaplan D, ed. Handbook of quantitative methodology for the social sciences. Newbury Park: Sage Publications; 2004:345-368.

19. Jun HJ, Corliss HL, Nichols LP, Pazaris MJ, Spiegelman D, Austin SB. Adult BMI trajectories and sexual orientation. Am J Prev Med. 2012;4:348-354.

20. Nylund KL, Asparouhov T, Muthén B. Deciding on the number of classes in latent class analysis and growth mixture modeling. A Monte Carlo simulation study. Struct Equ Model. 2007;14:535-569.

21. Tofighi D, Enders CK. Identifying the correct number of classes in growth mixture models. In: Hancock GR, Samuelsen KM, eds. Advances in latent variable mixture models. Greenwich, CT: Information Age. 2008;317-341.

22. Maguen S, Ren L, Bosch J, Marmar CR, Seal KH. Gender differences in mental health diagnoses among Iraq and Afghanistan veterans enrolled in VA healthcare. Am J Public Health. 2010;12:2450-2456.
23. Seal KH, Metzler TJ, Gima C, Bertenthal D, Maguen S, Marmar CR. Trends and risk factors for mental health diagnoses among Iraq and Afghanistan veterans using department of Veterans Affairs health care, 2002-2007. Am J Public Health. 2009;9:1651-1658.

24. Muthén LK, Muthén Bo. Mplus User's Guide. Sixth Edition. Los Angeles, CA: Muthén \& Muthén; 1998-2011.

25. Maguen S, Madden E, Cohen BE, Bertenthal D, Seal KH. Time to treatment among veterans of conflicts in Iraq and Afghanistan with psychiatric diagnoses. Psychiatr Serv. 2012;63(12):1206-1212.

26. Botoseneanu A, Liang J. Social stratification of body weight trajectory in middle-age and older Americans: results from a 14-year longitudinal study. J Aging Health. 2011;23:454-480.

27. Daubenmier $\mathbf{J}$, Kristeller $\mathbf{J}$, Hecht $\mathbf{F M}$, Maninger $\mathbf{N}$, Kuwata $\mathbf{M}$, Jhaveri $\mathbf{K}$, et al. Mindfulness Intervention for Stress Eating to Reduce Cortisol and Abdominal Fat among Overweight and Obese Women: An Exploratory Randomized Controlled Study. J Obes. 2011;2011:651936.

28. Hays NP, Roberts SB. Aspects of eating behaviors "disinhibition" and "restraint" are related to weight gain and BMI in women. Obesity. 2008; 1:52-58.

29. Fulton JJ, Lavender JM, Tull MT, Klein AS, Muehlenkamp JJ, Gratz KL. The relationship between anxiety sensitivity and disordered eating: the mediating role of experiential avoidance. Eat Behav. 2012;2:166169. Epub 2012 Jan 4

30. McLean CP, Miller NA, Hope DA. Mediating social anxiety and disordered eating: the role of expressive suppression. Eat Disord. 2007;1:41-54.

31. Seal KH, Maguen S, Cohen B, Gima KS, Metzler TJ, Ren L, et al. VA mental health services utilization in Iraq and Afghanistan veterans in the first year of receiving new mental health diagnoses. J Traum Stress. 2010;23:5-16.

32. Seal KH, Abadjian L, McCamish N, Shi Y, Tarasovsky T, Weingardt K. A randomized controlled trial of telephone motivational interviewing to enhance mental health treatment engagement in Iraq and Afghanistan veterans. Gen Hosp Psychiatry. 2012;34(5):450-459.

33. Yano EM, Hayes P, Wright S, Schnurr PP, Lipson L, Bean-Mayberry B, et al. Integration of women veterans into VA quality improvement research efforts: what researchers need to know. J Gen Intern Med. 2010;25(Suppl 1):56-61. 